\title{
RIQUEZA Y ABUNDANCIA DE LA MACROFAUNA EPIGEA EN CUATRO SISTEMAS TROPICALES DEL ESTADO DE CHIAPAS, MÉXICO
}

\section{RICHNESS AND ABUNDANCE OF EPIGEAL MACROFAUNA IN FOUR TROPICAL SYSTEMS OF CHIAPAS, MEXICO}

\section{RubÉn Francisco MANCILla -BRINDIS, ${ }^{1}$ Luis José RANGEL RUIZ, ${ }^{1, *}$ Armando FALCÓN-BRINDIS, ${ }^{2}$ JAQuelina GAMBOA AGUILAR ${ }^{1}$ y JUAn de Dios VALDEZ LEAL ${ }^{1}$}

\author{
${ }^{1}$ Laboratorio de Malacología. Universidad Juárez Autónoma de Tabasco. Carretera Villahermosa-Cárdenas Km. \\ 0.5 entronque con Saloya, C. P. 94250, Villahermosa, Tabasco, México Tel. Fax (993) 354-43 08. \\ ${ }^{2}$ Centro de Investigaciones Biológicas del Noroeste S.C. Av. Instituto Politécnico Nacional 195, Playa Palo \\ de Santa Rita Sur, La Paz, B.C.S. México; C.P. 23096. (RFMB) < franciscobrindis427@gmail.com>; (LJRR) \\ <ljrangel@msn.com>; (AFB) <armandofalcon14@hotmail.com>; (JGA)<jgamboaa@msn.com>; (JDVL) \\ $<$ jdvaldezleal@yahoo.com.mx>. \\ * Autor para correspondencia: <ljrangel@msn.com>. \\ Recibido: 08/11/2016; aceptado: 10/07/2017. \\ Editor responsable: Magdalena Cruz.
}

\begin{abstract}
Mancilla-Brindis, R. F., Rangel-Ruiz, L. J., Falcón-Brindis, A., Gamboa-Aguilar, J. y Valdez-Leal, J. D. (2017). Riqueza y abundancia de la macrofauna epigea en cuatro sistemas tropicales del Estado de Chiapas, México. Acta Zoológica Mexicana (n.s.), 33(3), 464-471.
\end{abstract}

RESUMEN. Se realizó un muestreo en cuatro sistemas vegetales con diferente grado de perturbación, (i.e. selva tropical, vegetación secundaria madura, vegetación secundaria joven y un cultivo agrícola) al norte de Chiapas, México. El objetivo de este estudio fue determinar las diferencias en la composición y estructura de las comunidades de macroinvertebrados presentes en la hojarasca. Para ello, se estimaron datos de abundancia $(\mathrm{N})$, riqueza especifica (S), diversidad de Shannon $\left(\mathrm{H}^{\prime}\right)$, equidad de Pielou (J) y el índice de similitud de Morisita-Horn. En cada sistema se tomaron 20 muestras colocadas al azar por medio de cuadrantes de $1 \mathrm{~m}^{2}$. Se recolectó la hojarasca y materia orgánica (humus) del cuadrante hasta una profundidad de $5.0 \mathrm{~cm}$, con la finalidad de colectar únicamente a los macroinvertebrados epigeos. En total se obtuvieron 1,259 especímenes, clasificados en tres phyla, nueve clases, 26 órdenes, 61 familias, 17 géneros y 100 morfotipos. La mayor $\mathrm{N}$ y $\mathrm{S}$ se encontraron en la selva, sin embargo, la mayor diversidad $\left(\mathrm{H}^{\prime}\right)$ ocurrió en la vegetación secundaria en etapa inicial de sucesión. El cultivo agrícola se mostró como el sistema con mayor equidad. Se observó una relación positiva entre el grado de conservación de los sistemas con la abundancia de los macroinvertebrados, pero no siempre con la riqueza de especies.

Palabras clave: Macroinvertebrados, comunidades, perturbación, sucesión vegetal.
Mancilla-Brindis, R. F., Rangel-Ruiz, L. J., Falcón-Brindis, A., Gamboa-Aguilar, J., \& Valdez-Leal, J. D. (2017). Richness and abundance of epigeal macrofauna in four tropical systems of Chiapas, Mexico. Acta Zoológica Mexicana (n.s.), 33(3), 464-471.

ABSTRACT. We sampled four vegetation systems with different degree of disturbance (i. e. tropical forest, mature and young secondary forest, and a cornfield) in northern Chiapas, Mexico. The objective of this study was to determine the differences in the composition and structure of macroinvertebrate communities in litter. We estimated abundance $(\mathrm{N})$, species richness $(\mathrm{S})$, Shannon's diversity $\left(\mathrm{H}^{\prime}\right)$, Pielou's evenness (J), and Morisita-Horn's similarity index. Twenty samples were randomly placed in each system by using 1-m2 quadrats. Leaf litter and organic matter (humus) were collected from the quadrat to a depth of $5.0 \mathrm{~cm}$, with the purpose of collecting only the epigeous macroinvertebrates. We collected 1,259 specimens from three phyla, nine classes, 26 orders, 61 families, 17 genera, and 100 morphotypes. The highest $\mathrm{N}$ and $\mathrm{S}$ were observed in the tropical forest, however, the highest diversity $\left(\mathrm{H}^{\prime}\right)$ occurred in the young secondary forests. The cornfield showed the highest evenness. There was a positive relation between the systems' conservation level and macroinvertebrate abundance, but not with species richness.

Key words: macroinvertebrates, communities, disturbance, plant succession. 


\section{INTRODUCCIÓN}

La fragmentación de los ecosistemas terrestres por la introducción de sistemas de cultivo, plantaciones forestales y pastizales ha sido una problemática ampliamente abordada (Cavelier \& Etter, 1995; Van der Hammen, 1995; Coral \& Bonilla, 2002; Cabrera-Dávila et al., 2007). El uso no sostenible y las constantes perturbaciones tienen un efecto negativo sobre la macrofauna epigea que habita en la superficie, alterando importantes procesos bióticos que mantienen el equilibrio edáfico (Feijoo et al., 1999).

La macrofauna epigea incluye aquellos artrópodos, moluscos y anélidos que se desarrollan debajo la hojarasca muerta del suelo. Su diámetro promedio es de 2 a $20 \mathrm{~mm}$. Los representantes más comunes incluyen algunos grupos de Coleoptera (escarabajos), Blattodea (cucarachas), Hymenoptera (hormigas), Mollusca (caracoles), Hemiptera (chinches), Arachnida (arañas, ácaros, esquizómidos y pseudoescorpiones), Isopoda (cochinillas) y Miriapoda (cien y mil pies) (Cabrera-Dávila et al., 2011a).

Una de sus funciones ecológicas es la reducción del tamaño de las partículas como primer paso en la desintegración de la materia orgánica, previo a la descomposición y reincorporación de nutrientes al suelo (Lavelle et al., 1992; Barajas-Guzmán \& Álvarez-Sánchez, 2003). Debido a que se encuentran en un estrato vertical inicial y poseen escaza vagilidad, las comunidades de fauna epigea responden ante los efectos de la fragmentación de hábitat, pérdida de cobertura vegetal y alteración del horizonte orgánico del suelo, ocasionadas por la transformación de ecosistemas por causas naturales o por el hombre (Fragoso et al., 1997).

En México se han realizado investigaciones acerca de las comunidades de la macrofauna del suelo en diferentes sistemas de uso de la tierra (Brown et al., 2001; 2004; García et al., 2009; Morales-Vásquez et al., 2014), destacando la importancia de su conservación en la medida que ayudan a mantener los procesos bióticos y por ende, la estabilidad del suelo y la vida que sobre éste se desarrolla (Huerta-Lwanga et al., 2008; Velásquez et al., 2009; Cabrera-Dávila et al., 2011b; Cabrera-Dávila, 2014).

Estas características de sensibilidad ante las perturbaciones y fragmentación les han conferido un potencial como indicadores de la calidad del hábitat (Lavelle et al., 1994a; Benckiser, 1997; Pérez et al., 2007; Bignell et al., 2012; Rousseau et al., 2013). En este trabajo, el objetivo fue analizar las diferencias en la composición y estructura de la comunidad de macrofauna presente en la hojarasca en cuatro sistemas vegetales, con diferente grado de perturbación, mediante el uso de parámetros ecológicos, esperando encontrar un patrón biológico que facilite la comprensión de los efectos de la fragmentación en hábitats tropicales del sureste de México.

\section{MATERIALES Y MÉTODOS}

Área de estudio. El área de estudio está integrada por parches de vegetación que corresponden a selva tropical, vegetación secundaria (acahuales) zonas agrícolas y ganaderas, dedicadas a la producción de ganado bovino (INEGI, 2013). El estudio se realizó en cuatro sistemas vegetales distribuidos en dos rancherías (R. Santa Teresa 3ra Sección y R. Hidalgo) en el municipio de Juárez Chiapas, cerca de las colindancias con el estado de Tabasco (Fig. 1).

En esta zona, el clima es de tipo cálido húmedo con lluvias casi todo el año (Am). La temperatura promedio anual es de $35^{\circ} \mathrm{C}$ y precipitaciones del orden de los 1,9002,000 mm (García, 1981). El relieve está conformado por lomeríos con pendientes suaves a moderadas cuya geología está dominada por rocas sedimentarias con suelos de tipo luvisol. La hidrología superficial posee escurrimientos permanentes (i.e. Arroyo Nuevo Mundo y Tepaté) e intermitentes (INEGI, 2013).

Las muestras se obtuvieron de cuatro sistemas vegetales ordenados de mayor a menor grado de conservación: selva tropical $(\mathrm{S})$, vegetación secundaria madura o acahual maduro (AM), con un periodo de regeneración aproximado de 20 años, acahual joven (AJ) con 10 años de sucesión y un cultivo de Zea mays L. de temporal (M) en segundo ciclo cosecha, cada sistema conformado por un área de 2 ha. La edad de los sistemas se obtuvo a través de consultas con los propietarios de los predios, quienes tienen registros de la edad de dichos sistemas con base al manejo. Debido a que cada año la zona está sujeta a inundaciones durante la época húmeda, el muestreo se efectuó durante marzo de 2013, al final de la estación lluviosa (Menéndez \& Cabrera-Dávila, 2014). Se realizó un solo muestreo puesto que el presente trabajo fue diseñado para contrastar los efectos de la fragmentación y disturbio entre hábitats y no para hacer comparaciones temporales o estacionales.

Se colectaron 20 muestras en cada sistema vegetal, donde cada una consistió en un cuadrante de $1 \mathrm{~m}^{2}$ colocado al azar, distanciados a $20 \mathrm{~m}$ entre sí, (Pérez et al., 1996; Correa-Sandoval et al., 1998; de la Torre, 2012). Se recolectó la hojarasca y materia orgánica (humus) del 


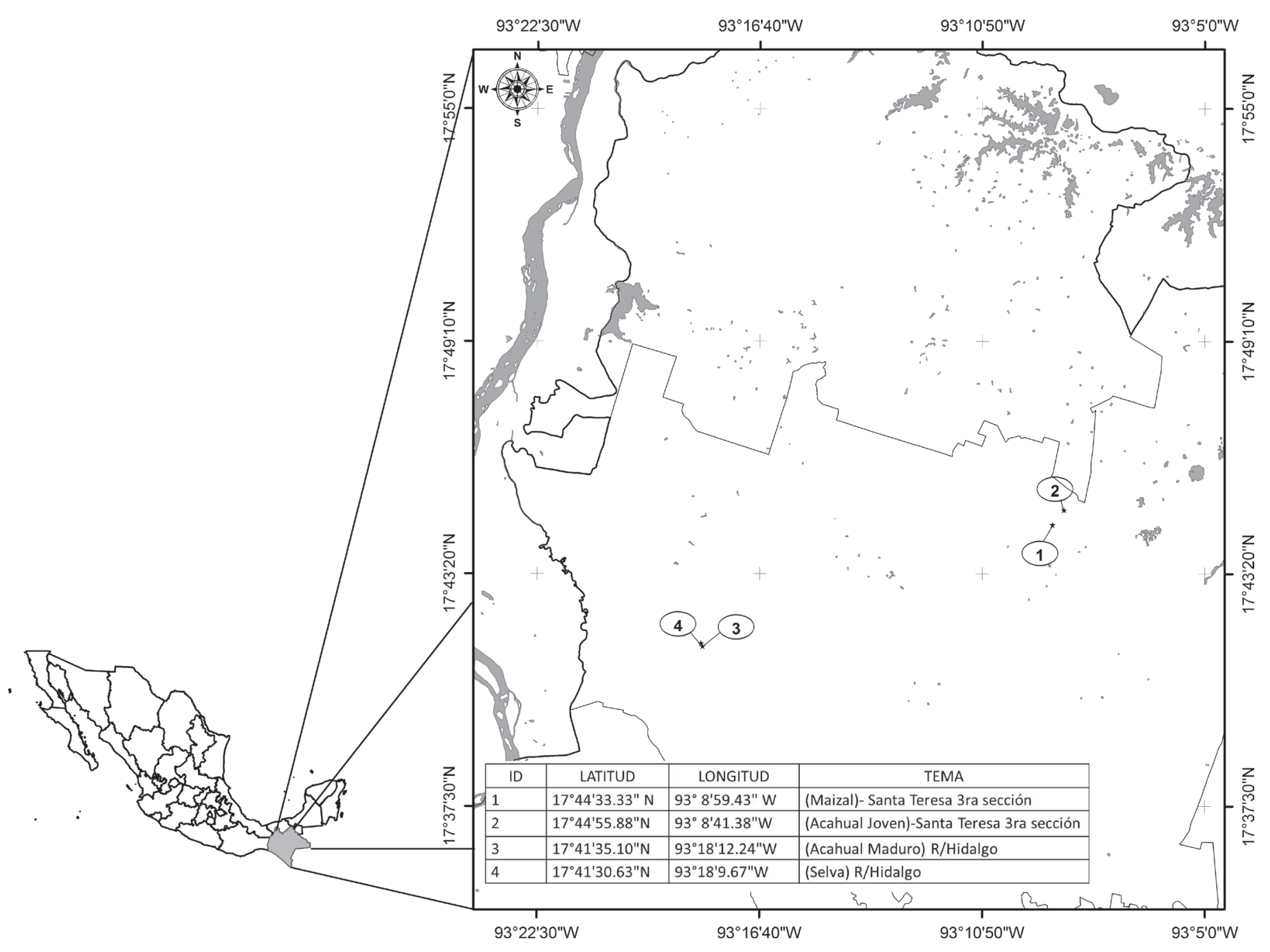

Figura 1. Área de estudio de los cuatro sistemas vegetales en Chiapas, México.

cuadrante hasta una profundidad de $5.0 \mathrm{~cm}$ (Rangel-Ruiz \& Gamboa-Aguilar, 2006; Oviedo \& de la Torre, 2014). Con la finalidad de colectar únicamente a los macroinvertebrados epigeos, los organismos fueron separados mediante tres tamices con aberturas de malla de $0.25,0.50$ y $1.00 \mathrm{~mm}$.

Posteriormente fueron llevados al laboratorio donde los organismos con exoesqueleto quitinoso fueron fijados con solución de alcohol al 70\% y glicerina al 5\%, para los anélidos se utilizó formol al 5\% y los moluscos se preservaron en solución de Railliet-Henry (930 ml de solución salina, $50 \mathrm{ml}$ de Formaldehido y $20 \mathrm{ml}$ de Ácido Acético) (Bignell et al., 2012).

Todos los organismos se identificaron a nivel de clase y orden, posteriormente se realizó una separación y cuantificación de los morfotipos presentes en cada orden y estos fueron sustitutos de las especies y utilizados para la determinación de los índices ecológicos (Morales \& Sarmiento, 2002).

Para la identificación taxonómica se utilizaron claves de identificación generales a nivel de orden (Brusca \& Brusca, 2003; Borror, 2005; Ubik et al., 2005).

Para la determinación de la estructura de la comunidad se calculó la abundancia $(\mathrm{N})$, riqueza específica $(\mathrm{S})$, diversidad de Shannon $\left(\mathrm{H}^{\prime}\right)$ y equidad de Pielou (J) (Krebs, 1999; Magurran, 2006). La similitud entre las comunidades de macroinvertebrados en los cuatro sistemas se estimó con el índice de Morisita-Horn. Los datos fueron analizados con el programa EstimateS Win900 (Colwell, 2012) y Multi-Variate Statistical Package MVSP 3.1. De- 
bido a que los datos no cumplen con los requerimientos de normalidad, se realizó un análisis no paramétrico de Kruskall-Wallis, donde se contrastaron hipótesis estadísticas de las respuestas de riqueza y abundancia de la fauna epigea en los cuatro sistemas vegetales (R Core Team, 2016).

\section{RESULTADOS}

Composición. La composición de los macroinvertebrados estuvo integrada por un total de 1,259 organismos que abarcaron a tres Phyla: Arthropoda (79 morfotipos), Mollusca (20 morfotipos) y Annelida (un morfotipo). La clase que presentó el mayor número de órdenes fue Insecta (Blattodea, Coleoptera, Hemiptera, Hymenoptera, Diplura y Lepidoptera), seguida de Arachnida (Acari, Schizomida, Araneae, Opiliones y Pseudoscorpiones), Diplopoda (Polyzoniida, Stemmiulida, Spirostreptida y Polydesmida), Gastropoda (Archaeogastropoda, Stylommatophora, Basommatophora y Systellommatophora); Chilopoda (Lithobiomorpha, Scolopendromorpha y Geophilomorpha) y un orden en cada una de las clases Clitellata (Haplotaxida), Entognatha (Diplura), Malacostraca (Isopoda) y Symphyla (no identificado). Lo anterior quedó agrupado en nueve clases y 26 órdenes en los cuatro sistemas vegetales. Los órdenes que presentaron mayor número de morfotipos fueron Coleoptera (25), Stylommatophora (17), Hymenoptera (9), Araneae (7), Acari (6) y Hemiptera (6).

Abundancia. Las clases más abundantes en los cuatro sistemas vegetales fueron Gastropoda $(n=778)$, Insecta $(n=$ $158)$ y Arachnida $(n=102)$. Sin embargo, Gastropoda fue la más numerosa en tres de estos sistemas, excepto en el acahual joven, el cual estuvo dominado por Insecta. En la selva, la clase Gastropoda presentó una abundancia relativa de $(79.58 \%)$, seguida por Insecta y Arachnida (6.62\% y $5.49 \%$ respectivamente). En el acahual maduro prevaleció la Gastropoda (51.20\%) como la clase más dominante, seguida por Insecta (17.74\%) y Arachnida (11.29\%). En el acahual joven se pudo observar una distribución más equitativa en las abundancias de Insecta $(24.10 \%)$, Gastropoda (20.51\%) y Diplopoda (16.41\%). En el cultivo de maíz ocurrió una elevada abundancia de Gastropoda (47.91\%), seguida por Insecta y Arachnida (20.83\% y $11.45 \%$ ) (Cuadro 1). Sin embargo, no se encontraron diferencias significativas entre las abundancias totales de las clases en todos los sistemas vegetales estudiados $(K=$ 3.8494, $p=0.2781$ ).

La mayor abundancia de macroinvertebrados se encontró en la selva $(\mathrm{n}=710)$, mientras que la menor en el cultivo de maíz ( $\mathrm{n}=96)$ (Cuadro 3 ).

De los 26 órdenes registrados, 14 ocurrieron en todos los sistemas vegetales, mismos que mostraron las mayores abundancias. Los órdenes Spirostreptida, Schizomida y Blattodea presentaron afinidad por la selva, Stemmiulida afinidad en el acahual maduro y Systellommatophora, Scolopendromorpha en el acahual joven. A pesar de esto, no presentaron diferencias significativas la abundancia total de los órdenes en los cuatro sistemas vegetales ( $K=$ 1.5426, $p=0.6724$ ). (Cuadro 2).

Índices Ecológicos. La mayor riqueza de morfotipos se presentó en la selva, mientras que la mínima prevaleció en el cultivo de maíz. De acuerdo al índice de diversidad de Shannon, el mayor valor se registró en el acahual joven $\left(\mathrm{H}^{\prime}=3.34\right)$ y el menor en la selva $\left(\mathrm{H}^{\prime}=1.86\right)$. Respecto a

Cuadro 1. Abundancia total de cada clase en los sistemas vegetales estudiados en Juárez, Chiapas. La abundancia relativa se muestra entre paréntesis. $\mathrm{S}=$ selva, $\mathrm{AM}=$ acahual maduro, $\mathrm{AJ}=$ acahual joven, $\mathrm{M}=$ maizal.

\begin{tabular}{|c|c|c|c|c|c|c|c|c|}
\hline \multirow{2}{*}{$\begin{array}{l}\text { Clase } \\
\text { Gastropoda }\end{array}$} & \multicolumn{2}{|c|}{$\mathrm{S}$} & \multicolumn{2}{|c|}{$\mathrm{AM}$} & \multicolumn{2}{|c|}{$\mathrm{AJ}$} & \multicolumn{2}{|c|}{$\mathrm{M}$} \\
\hline & 565 & (79.58) & 127 & $(51.20)$ & 40 & $(20.51)$ & 46 & $(47.91)$ \\
\hline Clitellata & 10 & $(1.41)$ & 15 & $(6.04)$ & 20 & $(10.25)$ & 6 & $(6.25)$ \\
\hline Malacostraca & 10 & $(1.41)$ & 2 & $(0.80)$ & 2 & $(1.02)$ & 4 & $(4.16)$ \\
\hline Insecta & 47 & $(6.62)$ & 44 & (17.74) & 47 & $(24.10)$ & 20 & $(20.83)$ \\
\hline Symphyla & 13 & $(1.83)$ & 7 & $(2.82)$ & 18 & $(9.23)$ & 4 & $(4.16)$ \\
\hline Entognatha & & & & & 5 & $(2.56)$ & & \\
\hline Total & 710 & & 248 & & 197 & & 96 & \\
\hline
\end{tabular}


Cuadro 2. Abundancia total de cada orden en los sistemas vegetales estudiados en Juárez, Chiapas. La abundancia relativa se muestra entre paréntesis. $\mathrm{S}=$ selva, $\mathrm{AM}=$ acahual maduro, $\mathrm{AJ}=$ acahual joven, $\mathrm{M}=$ maizal.

\begin{tabular}{|c|c|c|c|c|c|c|c|c|}
\hline Orden & & & & & & & & \\
\hline Stylommatophora & 554 & $(77.81)$ & 127 & $(51.21)$ & 38 & (19.29) & 45 & (46.88) \\
\hline Coleoptera & 32 & $(4.49)$ & 30 & $(12.1)$ & 22 & (11.17) & 6 & $(6.25)$ \\
\hline Acari & 29 & $(4.07)$ & 2 & $(0.81)$ & 13 & $(6.6)$ & 2 & (2.08) \\
\hline S/O (C:Symphyla) & 13 & $(1.83)$ & 7 & $(2.82)$ & 18 & $(9.14)$ & 4 & $(4.17)$ \\
\hline Polydesmida & 12 & $(1.69)$ & 19 & (7.66) & 32 & $(16.24)$ & 2 & $(2.08)$ \\
\hline Haplotaxida & 10 & (1.4) & 15 & $(6.05)$ & 20 & $(10.15)$ & 6 & $(6.25)$ \\
\hline Isopoda & 10 & (1.4) & 2 & $(0.81)$ & 2 & $(1.02)$ & 4 & (4.17) \\
\hline Geophilomorpha & 7 & $(0.98)$ & 4 & (1.61) & 1 & $(0.51)$ & 3 & (3.13) \\
\hline Hemiptera & 7 & $(0.98)$ & 9 & (3.63) & 4 & $(2.03)$ & 6 & $(6.25)$ \\
\hline Araneae & 6 & $(0.84)$ & 14 & $(5.65)$ & 7 & (3.55) & 5 & $(5.21)$ \\
\hline Hymenoptera & 6 & $(0.84)$ & 1 & $(0.4)$ & 12 & (6.09) & 2 & (2.08) \\
\hline Diplura & 1 & $(0.14)$ & 4 & (1.61) & 12 & (6.09) & 5 & $(5.21)$ \\
\hline Opiliones & 1 & $(0.14)$ & 1 & $(0.4)$ & 1 & $(0.51)$ & 2 & $(2.08)$ \\
\hline Pseudoscorpiones & 1 & $(0.14)$ & 11 & (4.44) & 3 & $(1.52)$ & 2 & (2.08) \\
\hline Spirostreptida & 1 & $(0.14)$ & & & & & & \\
\hline Schizomida & 2 & $(0.28)$ & & & & & & \\
\hline Blattodea & 1 & $(0.14)$ & & & & & & \\
\hline Stemmiulida & & & 1 & $(0.4)$ & & & & \\
\hline Systellommatophora & & & & & 1 & $(0.51)$ & & \\
\hline Scolopendromorpha & & & & & 6 & $(3.05)$ & & \\
\hline Polyzoniida & 3 & $(0.42)$ & 1 & $(0.4)$ & & & & \\
\hline Basommatophora & 10 & $(1.4)$ & & & 1 & $(0.51)$ & & \\
\hline Lithobiomorpha & 3 & $(0.42)$ & & & 2 & $(1.02)$ & & \\
\hline Archaeogastropoda & 1 & $(0.14)$ & & & & & 1 & $(1.04)$ \\
\hline Lepidoptera & & & & & 2 & $(1.02)$ & 1 & (1.04) \\
\hline
\end{tabular}

la equidad de Pielou, el maizal se presentó como el más equitativo $(\mathrm{J}=0.86)$ y la selva como el ambiente con menor equidad $(\mathrm{J}=0.46)$ (Cuadro 3).

Al comparar la composición de morfotipos de fauna epigea entre las cuatro comunidades vegetales, la selva y el acahual maduro presentaron la mayor similitud (0.612), seguidos por el acahual joven y el maizal (0.297) (Fig. 2).

\section{DISCUSIÓN}

La abundancia total en cuanto a la clase y orden de la macrofauna de cada sistema vegetal, no mostró diferencias significativas, es decir, se siguió conservando la misma relación morfotipo-número de individuos. Este mismo efecto se ha observado en otros sistemas vegetales con distinto grado de fragmentación y sucesión como en los páramos venezolanos (Morales \& Sarmiento, 2002). De acuerdo a Kogan (1981), este comportamiento puede atribuirse a que en algunos casos la perturbación del hábitat favorece a ciertas especies de estrategas " $r$ " capaces de usar los nuevos recursos disponibles y recolonizar rápidamente el área afectada.

Cuadro 3. Valores de abundancia $(\mathrm{N})$, riqueza específica $(\mathrm{S})$, diversidad de Shannon $\left(\mathrm{H}^{\prime}\right)$, equidad de Pielou (J), en los cuatro sistemas vegetales estudiados. $\mathrm{S}=$ selva, $\mathrm{AM}=$ acahual maduro, $\mathrm{AJ}=$ acahual joven, $\mathrm{M}=$ maizal .

\begin{tabular}{lllll}
\hline & $\mathrm{S}$ & AM & AJ & M \\
\hline $\mathrm{N}$ & 710 & 248 & 197 & 96 \\
$\mathrm{~S}$ & 55 & 37 & 50 & 37 \\
$\mathrm{H}^{\prime}$ & 1.86 & 2.67 & 3.34 & 3.12 \\
$\mathrm{~J}$ & 0.46 & 0.73 & 0.85 & 0.86 \\
\hline
\end{tabular}




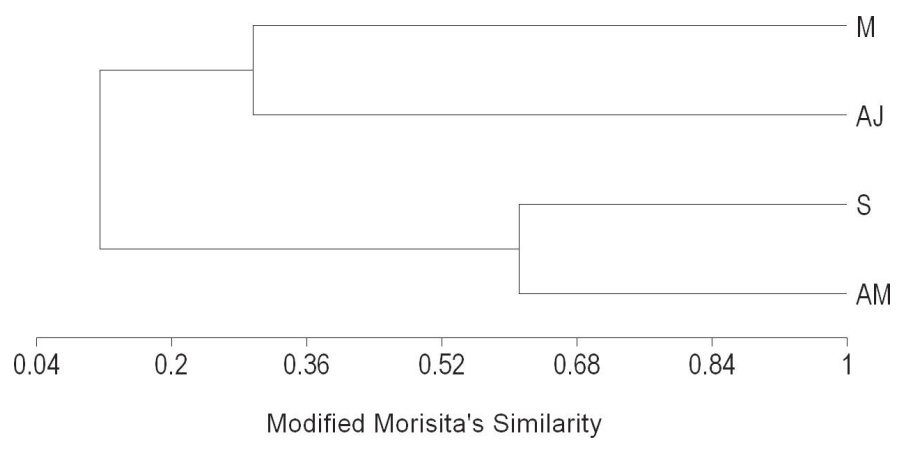

Figura 2. Dendograma de similitud de Morisita-Horn de las comunidades de fauna epigea en cuatro sistemas vegetales en el Municipio de Juárez Chiapas. $\mathrm{S}=$ selva, $\mathrm{AM}=$ acahual maduro, $\mathrm{AJ}=$ acahual joven, $\mathrm{M}=$ maizal.

La clase Diplopoda y Arachnida han sido mencionadas como indicadoras de ambientes conservados (BuenoVillegas \& Rojas, 1999; Ruiz-Cobo et al., 2010). Estos taxa estuvieron presentes y en abundancias similares tanto en la selva, como aquellos en proceso de sucesión. No obstante, en la zona de cultivo estos grupos estuvieron ausentes. Lo anterior coincide con Ryszkowski (1985), quien señala a los miriápodos (diplópodos) como organismos muy sensibles a los efectos de la fragmentación de hábitat provocado por las actividades agrícolas.

Las selvas tropicales generalmente ofrecen abundante cobertura vegetal arbórea, lo cual disminuye la entrada de luz solar, e impide el sobrecalentamiento de los estratos inferiores (Naranjo \& Sánchez, 2003), esta situación, mantiene niveles de humedad elevados $(>80 \%)$ y permite formar un microclima que además de ofrecer alimento, protege a los artrópodos epígeos de cuerpos blandos, así como a los moluscos (Neher, 1999; Rangel-Ruiz \& Gamboa-Aguilar, 2001). Lo anterior permite esperar resultados coherentes en este trabajo, lo cual se vio reflejado en una mayor abundancia de organismos, especialmente de gastrópodos en los sistemas con mayor grado de conservación y etapas avanzadas de sucesión. Otros estudios realizados en selvas tropicales de Tabasco, México (Rangel-Ruiz et al., 2004; Rangel-Ruiz \& Gamboa-Aguilar, 2006), señalan a la clase Gastropoda como un grupo más diverso y abundante que aprovechan los microambientes formados a partir de la alta humedad y temperatura moderada durante la mayor parte del año.

Los valores de diversidad fueron bajos para la selva y de medios a altos para el acahual maduro, acahual joven y maizal. De acuerdo a la clasificación de Ramírez (2006), los valores de $\mathrm{H}^{\prime}$ que fluctúan entre 2.5 y 3.9 son frecuen- tes en ambientes poco alterados o procedentes de matrices agropecuarias que presentan una estructura heterogénea. No obstante, el bajo valor de $\mathrm{H}^{\prime}$ (1.86) en la Selva no coincide con el grado de ambiente poco alterado, y se atribuye a su baja equidad (mayor dominancia), más que a su riqueza de especies/morfotipos (Magurran, 2006).

Por su parte, Ochoa-Gaona et al. (2007) señalan que la vegetación secundaria (acahuales) representa una fase intermedia que propicia alta riqueza y donde se alternan especies de hábitats perturbados y especies propias de ambientes conservados. Sin embargo, el valor alto en el maizal, podría deberse a la presencia de abundante materia orgánica producto del deshierbe y el esquilmo de la cosecha anterior, lo cual puede propiciar alimento y refugio para la macrofauna. Cabe agregar que este sistema colindaba con una zona inundable cubierta de vegetación acuática lo que podría explicar la gran diversidad y abundancia de moluscos del orden Stylommatophora. De manera semejante, Morales-Vásquez et al. (2014) obtuvieron un valor alto de $\mathrm{H}^{\prime}$ en un cultivo de maíz explicando que el resultado podría deberse al efecto del manejo pos cosecha, donde se permitió la incorporación de abono orgánico y hojarasca en un cultivo de riego.

La mayor similitud entre la selva y el acahual maduro se debe a que el primero se trata de un sistema no alterado y el segundo se encuentra en la mayor etapa de recuperación o proceso sucesional (Collins, 1980). Ambos sistemas se asemejan al presentar una abundante capa de materia orgánica en suelo que favorece el desarrollo de macroinvertebrados. Por su parte, la similitud encontrada entre los sistemas vegetales alterados como el maizal y el acahual joven eran de esperarse. Aunque ambos ambientes se encuentran bajo una dinámica de sucesión distinta, el arreglo de comunidades de macroinvertebrados conserva mayor similitud comparado con un ambiente climax o en sucesión avanzada (Botina et al., 2012).

En este trabajo, la ausencia de los morfotipos de los órdenes Spirostreptida, Schizomida, y Blattodea en sistemas alterados (maizal, acahual joven y acahual maduro), sugiere que podrían tratarse como grupos indicadores de estabilidad del ecosistema. Se ha señalado que estos organismos son sensibles a la fragmentación y alteración del hábitat debido su escasa vagilidad y especificidad en cuanto a los requerimientos microclimáticos (Pashanasi, 2001; Lavelle et al., 2003; Ruiz-Cobo et al., 2010; Velásquez et al., 2009; Cabrera-Dávila et al., 2011b). No obstante, se requiere realizar más estudios que ayuden a comprender la dinámica ecológica que ocurre con la fauna epigea en ambientes tropicales del sureste de México. 


\section{CONCLUSIONES}

En los sistemas vegetales tropicales del presente trabajo, los tres phyla más dominantes en la hojarasca son Arthropoda, Mollusca y Annelida. El arreglo de comunidades de fauna epigea es variable en función del nivel de sucesión vegetal y el uso de suelo a causa de actividades agropecuarias.

Se observó una relación entre la abundancia de organismos epígeos con el nivel de perturbación de los sistemas vegetales. El ambiente clímax (selva) tuvo casi un $700 \%$ más abundancia de organismos que el ambiente más degradado (cultivo). Sin embargo, la relación abundancia-riqueza fue similar en todos los sistemas.

AGRADECIMIENTOS. Los autores del presente estudio agradecen a los revisores anónimos que intervinieron en la mejora de este manuscrito.

\section{LITERATURA CITADA}

Barajas-Guzmán, G. \& Álvarez-Sánchez, J. (2003). La comunidad de desintegradores en una selva trópical húmeda. En: Ecología del Suelo en la Selva Tropical Húmeda de México (Eds. J. ÁlvarezSánchez y E. Naranjo-García). Instituto de Ecología, A.C. Instituto de Biología y Facultad de Ciencias. Universidad Autónoma de México (UNAM). Xalapa, México. p.162.

Benckiser, G. (1997). General Introduction. Pp. 1-6. In: G. Benckiser (ed.): Fauna in Soil Ecosystems Recycling Processes, Nutrient Fluxes and Agricultural Production. Marcel Dekker, Inc., New York.

Bignell, D. E., Constantino, R., Csuzdi, C., Karyanto, A., Konaté, S., Louzada, J., Susilo, F. X., Tondoh, J. E. \& Zanetti, R. (2012). Macrofauna. Pp. 91-92. In: M. S. Moreira, E. J. Huising, D. E. Bignell, editores. Manual de biología de suelos tropicales. Muestreo y caracterización de la biodiversidad bajo suelo. Instituto Nacional de Ecología.

Botina, G. B., Velásquez, I. A., Bacca, T., Castillo, F. J. \& Dias, G. L. (2012). Evaluación de la macrofauna del suelo en Solanum tuberosum (Solanales: Solanaceae) con sistemas de labranza tradicional y mínima. Boletín Científico. Centro de Museos. Museo de Historia Natural, 16(2), 69-77.

Brown, G., Fragoso, C., Barois, I., Rojas, P., Patrón, J., Bueno, J., Moreno, A., Lavelle, P., Ordaz, V. \& Rodríguez, C. (2001). Diversidad y rol funcional de la macrofauna edáfica en los ecosistemas tropicales mexicanos. Acta Zoológica Mexicana (n.s.), Numero especial 1, 79-110.

Brown, G., Moreno, A. G., Barois, I., Fragoso, C., Rojas, P., Hernández, B. \& Patrón, J. C. (2004). Soil macrofauna in SE Mexican pastures and the effect of conversion from native to introduced pastures. Agriculture, Ecosystems and Environment, 103, 313327.
Borror, D. J., Triplehorn, C. A., \& Johnson, N. F. (2005). An introduction to the study of insects. 7th Ed. Philadelphia, Saunders College Publishing. 865 p.

Brusca, R. \& Brusca, G. (2003). Invertebrates. Second Edition. USA. $966 \mathrm{p}$.

Bueno-Villegas, J. \& Rojas, F. P. (1999). Fauna de milpiés (Artrópoda: Diplopoda) edáficos de una selva alta de los Tuxtlas, Ver. México. Acta Zoológica Mexicana (n.s); 76, 59-83.

Correa-Sandoval, A., García-Cubas, A. \& Reguero, M. 1998. Gastrópodos terrestres de la región oriental de San Luis Potosí, México. Acta Zoológica Mexicana (n.s.), 73, 1-17.

Colwell, R. K., Chao, A., Gotelli, N. J., Lin, S. Y., Mao, C. X., Chazdon, R. L. \& Longino, J. T. (2012). Models and estimators linking individual-based and sample-based rarefaction, extrapolation, and comparison of assemblages. Journal of Plant Ecology, 5, 3-21.

Cabrera-Dávila, G. (2014). Manual práctico sobre la macrofauna edáfica como indicador biológico de la calidad del suelo, según resultados en Cuba. Pastos y Forrajes, 35(4), 349-364.

Cabrera-Dávila, G., Robaina, N. \& Ponce de León, D. (2011a). Composición funcional de la macrofauna edáfica en cuatro usos de la tierra en las provincias de Artemisa y Mayabeque, Cuba. Pastos y Forrajes, 34(2), 331-346.

Cabrera-Dávila, D. G., Robaina, N. \& Ponce de León, D. (2011b). Riqueza y abundancia de la macrofauna edáfica en cuatro usos de la tierra en las provincias de Artemisa y Mayabeque, Cuba. Pastos y Forrajes, 34, 313

Cabrera-Dávila, G., Martínez, M. A. \& Rodríguez, C. (2007). La macrofauna del suelo en sistemas agroecológicos en Cuba, Brenesia, 67, 45-57.

Cavelier, J. \& Etter, A. (1995). Deforestation of montane forest in Colombia as a result of illegal plantations of opium (Papaver somniferum). Pp 541-550. In: S. P. Churchill, H. Balslev, E. Forero \& J. L. Luteyn (eds). Biodiversity and conservation of Neotropical montane forests. The New York Botanical Garden, New York.

Collins, N. M. (1980). The distribution of soil macrofauna on the west ridge of Gunnung (mount) Mulu, Sarawak. Oencologia, 44: 263275.

Coral, D. \& Bonilla, C. (2002). Impacto de las prácticas agrícolas sobre la macrofauna del suelo en la cuenca alta del Lago Guamués, Pasto, Colombia. Suelos Ecuatoriales, 32, 157-160.

de la Torre, S. (2013). Research in Agricultural and Urban Areas in Galapagos: A Biological Perspective. Science and Conservation in the Galapagos Islands. Vol. 1, pp 185-198.

Feijoo, A., Knapp, E. B., Lavelle, P. \& Moreno, A. G. (1999). Quantifying soil macrofauna in a Colombian watershed, Pedobiologia, 43, 513-517.

Fragoso, C., Brown, G., Patrón, I. C., Blanchart, E. \& Lavelle, P. (1997). Agricultural intensification, soil biodiversity and agroecosystem function in the tropics: The role of earthworms. Applied of Soil Ecology, 6, 17-XX.

García J. A., Barois, I., Santos, M., Rojas, P., Fragoso, C., Moron, M. A., Bueno-Villegas, J. \& Sormani. C. (2009). Land use and diversity of the soil Macrofauna in Santa Marta, Los Tuxtlas, Veracruz, Mexico. Pp.135-190. In: Below-Ground Biodiversity in Sierra Santa Marta, Los Tuxtlas, Veracruz, México (Eds. Barois, I., E. J. Huising, P. Okoth, D. Trejo \& M. De Los Santos). Instituto 
de Ecología, A. C.

García, E. (1981). Modificaciones al Sistema de Clasificación Climática de Köppen. Instituto de Geografía, UNAM; México, D.F; $246 \mathrm{pp}$.

Huerta-Lwanga, E., Rodríguez-Olán, J., Evia-Castillo, I., Montejo-Meneses, E., Cruz-Mondragón, M. \& García-Hernández, R. (2008). Relación entre la fertilidad del suelo y su población de macroinvertebrados. Terra Latinoamericana, 26, 171-181.

INEGI: Instituto Nacional de Estadística y Geografía. 2013. Anuario Estadístico de Chiapas 2012. Aguascalientes, México. 622 p.

Kogan, M. (1981). Dynamics of insect adaptations to soybeans: Impact of integrated pest management. Environmental Entomology, 10, 663-670.

Krebs, C. J. (1999). Ecological Methodology, 2nd ed. Addison-Wesley Educational. 620 p.

Lavelle, P., Blanchart, E., Martin, A., Spain, A.V. \& Martin, S. (1992). Impact of soil fauna on the properties of soils in the Humid Tropics. In: Myths and Science of Soils of the Tropics. Soil Sci. Soc. Special Publication 29,157-XX

Lavelle, P., Dangerfield, M., Fragoso, C., Eschenbrenner, V., López-Hernández, D., Pashanasi, B. \& Brussaard, L. (1994a). The biological management of tropical soil fertility. Pp 137-169. In: P. L. Woomer \& M. J. Swift (Eds.). The relationship between soil macrofauna and tropical soil fertility, Wiley, Chichester.

Lavelle, P., Senapati, B. \& Barros, E. (2003). Soil macrofauna. Pp. 303-323. In: G. Schroth \& F. L. Sinclair (Eds.). Trees, crops and soil fertility. Concepts and research methods. CABF Publishing, UK.

Magurran, A. E. (2006). Measuring biological diversity. Blackwell Publishing, Malden, Massachusetts. $256 \mathrm{p}$.

Morales, J. \& Sarmiento, L. (2002). Dinámica de los macroinvertebrados edáficos y su relación con la vegetación en una sucesión secundaria en el páramo Venezolano. Ecotropicos, 15, 99-110.

Morales-Vásquez, E., R. S. Miguel-Méndez, A. Vázquez-Xochipa, J. Barrientos-Roldan, M. Gutiérrez-Carmona, \& C. Altamirano-Leal. (2014). Análisis de la diversidad de la macrofauna edáfica por estratos en dos usos de suelo en San Lorenzo Ometepec, Puebla. Entomología Mexicana, 1, 514-518.

Menéndez, Y. I. \& Cabrera-Dávila, G. (2014). La macrofauna de la hojarasca en dos sistemas con diferente uso de la tierra y actividad ganadera en Cuba. Revista Cubana de Ciencia Agrícola, 48, 2.

Neher, D. A. (1999). Soil community and ecosystem processes. Comparing agricultural ecosystems with natural ecosystems. Agroforestry Systems, 45, 159-185.

Oviedo, I. V., \& de la Torre, S. (2014). Estudio preliminar de caracoles terrestres en la Isla San Cristóbal, Galápagos. ACI Avances en Ciencias e Ingenierías, 6 (2). Pp?

Naranjo, G. E. \& Sánchez, J .A. (2003). Ecología del Suelo en la Selva Tropical Húmeda de México. Instituto de Biología, Facultad de Ciencias, UNAM. Xalapa; México. 316 p.

Ochoa-Gaona, S., Hernández-Vázquez, F., De Jong, B. H. G. \& Gurrí-García, F. D. (2007). Pérdida de diversidad florística ante un gradiente de intensificación del sistema agrícola de Roza-Tumba-Quema: un estudio de caso en la Selva Lacandona, Chiapas, México. Boletín de la Sociedad Botánica de México, 81, 65-80.

Pashanasi, B. (2001). Estudio cuantitativo de la macrofauna del suelo en diferentes sistemas de uso de la tierra en la Amazonia Peruana. Folia Amazónica, 12(1-2), 75-97.

Pérez, A. M., Villaseca, J. C. \& Zione. N. (1996). Sinecología básica de moluscos terrestres en cuatro formaciones vegetales de Cuba. Revista de Biología Tropical, 44, 133-146.

Pérez, A. M., Sotelo, M., Siria, I., Alkemade, R. \& Aburto, L. (2007). Developing a species based model for biodiversity assessment in an agricultural landscape in Nicaragua. Gaia, 8, 1-54.

Ramírez, G. A. (2006). Ecología: Métodos de muestreo y análisis de poblaciones y comunidades. Editorial Pontificia Universidad Javeriana, Bogotá, Bogotá, Colombia. 271 p.

Rangel-Ruiz, L. J. y J. Gamboa-Aguilar. (2001). Diversidad Malacológica en la Región Maya. I. Parque Estatal de la Sierra", Tabasco, México. Acta Zoológica Mexicana (n.s.) 82, 1-12.

Rangel-Ruiz, L. J., Gamboa-Aguilar, J. \& Alegría-Ruiz, F. (2004). Diversidad malacológica en la Región Maya. II. Parque Estatal Agua Blanca. Tabasco. México. Acta Zoológica Mexicana. (n.s), 20, 55-62.

Rangel-Ruiz, L. J. \& Gamboa-Aguilar, J. (2006). Listado preliminar de gasterópodos terrestres de "Boca del Cerro" Tenosique, Tabasco, México. Kuxulkab', 11: 51-57.

Rousseau, L., Fonte, S., Téllez, O., Hock, R., \& Lavelle, P. (2013). Soil macrofauna as indicators of soil quality and land use impacts in smallholder agroecosystems of western Nicaragua. Ecological Indicators, 27, 71-82.

Ruiz-Cobo, D. H., Feijoo, A. \& Rodríguez, C. (2010). Comunidades de macroinvertebrados edáficos en diferentes sistemas de uso de la tierra en la parte media de la cuenca del Río Otún, Colombia. Acta Zoológica Mexicana (n.s.) Número especial 2, 165-178.

Ryszkowski, L. (1985). Impoverishment of soil fauna due to agriculture. Intecol Bulletin 12, 7-17.

R Development Core Team. (2016). R: A Language and Environment for Statistical Computing. R Foundation for Statistical Computing, Vienna, Austria.

Ubik, D., Paquin, P., Cushing, P. E. \& Roth, V. (2005). Spiders of North America. American Arachnological Society.

Van der Hammen, T. (1995). Global change, biodiversity and conservation of Neotropical montane forests. Pp 603-607. In: S. Churchill, H. Balslev, E. Forero, J. Luteyn, (Eds.). Biodiversity and conservation of Neotropical montane forests. The New York Botanical Garden, New York.

Velásquez, E., Lavelle, P., Rendeiro, C., Martins, M., Barot, S. \& Grimaldi, M. (2009). Cambios en las comunidades de plantas influenciados por la macroagregación del suelo a través de las actividades de la macrofauna del suelo en la Amazonía brasileira. Disponible en: http://www.iamazonica.org.br/conteudo/ eventos/biodiversidadedesolo/pdf/Resumos/ Painel3_Velasquez E.pdf. (Revisado 04-02-2016). 